\title{
On rational quadratic differential forms
}

\author{
K. Takaba* H.L. Trentelman ${ }^{* *}$ J.C. Willems ${ }^{* * *}$ \\ * Department of Applied Mathematics and Physics \\ Graduate School of Informatics \\ Kyoto University, Kyoto 606-8501, Japan \\ (e-mail: takaba@amp.i.kyoto-u.ac.jp) \\ ** Mathematics Department, University of Groningen, P.O. Box 800, \\ 9700 AV Groningen, The Netherlands \\ (e-mail: h.l.trentelman@math.rug.nl) \\ *** ESAT SISTA, K.U. Leuven, Kasteepark Arenberg 10, Leuven \\ B-3001, Belgium (e-mail: Jan.Willems@esat.kuleuven.be)
}

\begin{abstract}
In linear system theory, we often encounter the situation of investigating some quadratic functionals which represent Lyapunov functions, energy storage, performance measures, e.t.c. Such a quadratic functional is called a quadratic differential form (QDF) in the context of the behavioral approach. In the past works, a QDF is usually defined in terms of a polynomial matrix. The contribution of this paper is to present a new and more general formulation of QDF's in terms of rational functions rather than polynomials. A QDF defined by rational functions is called a rational QDF. Unlike polynomial QDF's, a rational QDF defines a set of values of a quadratic functional. It turns out that several basic features of polynomial QDF's (nonnegativity, average nonnegativity, e.t.c.) can be generalized to the case of rational QDF's.
\end{abstract}

Keywords: linear systems, polynomial methods, quadratic differential form, rational representation

\section{INTRODUCTION}

In analysis and synthesis of dynamical systems, we often encounter the situation of investigating some functionals which represent Lyapunov functions, energy supply, energy storage, performance measures, and so forth. In particular, if the system under consideration is linear time-invariant, the functionals can be quadratic forms of the system variables and their derivatives. Such a quadratic form is called a quadratic differential form (QDF) which was introduced in the context of the behavioral system theory by Willems and Trentelman (1998).

In the behavioral framework, the QDF's have been playing a crucial role in many aspects of system and control theory: Lyapunov stability (Willems and Trentelman 1998, Peeters and Rapisarda 2001, Kojima and Takaba 2005), dissipation theory (Willems and Trentelman 1998, Willems and Trentelman 2002, Kaneko and Fujii 2000, 2003), linear quadratic optimal control (Willems and Valcher 2005), $\mathcal{H}^{\infty}$ control (Trentelman and Willems 1999, Willems and Trentelman 2002, Belur and Trentelman2004) and stability analysis of uncertain or nonlinear interconnections (Pendharkar Pillai 2007, Takaba 2005, Willems and Takaba 2007). Note that, in these past works, QDF's are usually defined in terms of polynomial matrices.

In this paper, as a generalization of the 'polynomial' QDF's described above, we will present a new formulation of a QDF in terms of a rational function, and will examine the basic features of such a type of QDF's. We call such a QDF defined by rational functions a rational $Q D F$. Note that the need for rational QDF's arises, for example, in the stability analysis of interconnected or feedback systems via rational multipliers or integral quadratic constraints (for the previous works on related topics, see e.g. Megretski and Rantzer 1997, Iwasaki and Hara 1998, e.t.c.). It will be shown that several basic features of polynomial QDF's such as nonnegativity and average nonnegativity can be generalized to the case of rational QDF's, and necessary and sufficient conditions for these features will be derived.

\section{Notations:}

$\mathbb{R}, \mathbb{C}$ : the fields of real numbers and complex numbers $i \mathbb{R}:=\{\lambda \in \mathbb{C} \mid \lambda=\mathrm{i} \omega, \omega \in \mathbb{R}\}$

$\mathbb{R}[\xi]$ : the ring of polynomials

$\mathbb{R}[\zeta, \eta]$ : the ring of two-variable polynomials

$\mathbb{R}(\xi)$ : the ring of rational functions

$\mathbb{R}(\zeta, \eta)$ : the ring of two-variable rational functions $\mathbb{R}^{\mathrm{p} \times \mathrm{q}}: \mathrm{p} \times \mathrm{q}$ real matrices

$\mathbb{R}^{\mathrm{p} \times \mathrm{q}}[\xi]: \mathrm{p} \times \mathrm{q}$ polynomial matrices

$\mathbb{R}^{\mathrm{p} \times \mathrm{q}}[\zeta, \eta]: \mathrm{p} \times \mathrm{q}$ two-variable polynomial matrices

$\mathbb{R}^{\mathrm{p} \times \mathrm{q}}(\xi): \mathrm{p} \times \mathrm{q}$ rational matrices

$\mathbb{R}^{\mathrm{p} \times \mathrm{q}}(\zeta, \eta): \mathrm{p} \times \mathrm{q}$ two-variable rational matrices

$\mathbb{R}_{\mathrm{S}}^{\mathrm{p} \times \mathrm{p}}: \mathrm{p} \times \mathrm{p}$ symmetric real matrices

$\mathbb{R}_{\mathrm{s}}^{\mathrm{p} \times \mathrm{p}}[\zeta, \eta]: \mathrm{p} \times \mathrm{p}$ symmetric two-variable polynomial matrices

$\mathbb{R}_{\mathrm{S}}^{\mathrm{p} \times \mathrm{p}}(\zeta, \eta): \mathrm{p} \times \mathrm{p}$ symmetric two-variable rational matrices $\mathfrak{C}^{\infty}\left(\mathbb{R}, \mathbb{R}^{\mathrm{w}}\right)$ : infinitely differentiable functions from $\mathbb{R}$ to $\mathbb{R}^{\mathrm{w}}$ $\mathfrak{D}$ : compact support functions

Note that we will often use "•" to denote irrelevant dimensions of a vector or a matrix.

For a two-variable polynomial or rational matrix $M(\zeta, \eta)$, we call it symmetric if it satisfies $M(\zeta, \eta)=M^{\top}(\eta, \zeta)$. 


\section{QUADRATIC DIFFERENTIAL FORM DEFINED BY A POLYNOMIAL MATRIX}

We first present the original definition of a QDF in terms of a two-variable polynomial matrix, and briefly review some basic features of QDF's from Willems and Trentelman (1998), Rapisarda and Willems (2004), Kaneko and Fujii (2004).

A quadratic differential form (QDF) $Q_{\Phi}$ is defined as a quadratic form of $w \in \mathfrak{C}^{\infty}\left(\mathbb{R}, \mathbb{R}^{\mathrm{w}}\right)$ and its derivatives:

$$
\begin{aligned}
Q_{\Phi}: \mathfrak{C}^{\infty}\left(\mathbb{R}, \mathbb{R}^{\mathrm{w}}\right) & \rightarrow \mathfrak{C}^{\infty}(\mathbb{R}, \mathbb{R}), \\
w & \mapsto Q_{\Phi}(w):=\sum_{i=0}^{k} \sum_{j=0}^{k}\left(\frac{d^{i} w}{d t^{i}}\right)^{\top} \Phi_{i j}\left(\frac{d^{j} w}{d t^{j}}\right),
\end{aligned}
$$

where $\Phi_{i j} \in \mathbb{R}^{\mathrm{w} \times \mathrm{w}}$ and $\Phi_{j i}^{\top}=\Phi_{i j}(i, j=0,1, \ldots, k)$. Throughout this paper, we will assume that all variables are $\mathfrak{C}^{\infty}$-functions of time $t$ for simplicity of discussion.

We can associate $Q_{\Phi}$ with a symmetric two-variable polynomial matrix

$$
\Phi(\zeta, \eta)=\sum_{i=0}^{k} \sum_{j=0}^{k} \zeta^{i} \eta^{j} \Phi_{i j} \in \mathbb{R}_{\mathrm{s}}^{\mathrm{w} \times \mathrm{w}}[\zeta, \eta] .
$$

Notice that the indeterminates $\zeta$ and $\eta$ correspond to the differentiation on $w^{\top}$ and $w$, respectively.

We consider the factorization of $\Phi \in \mathbb{R}_{\mathrm{s}}^{\mathrm{w} \times \mathrm{w}}[\zeta, \eta]$ in the form of

$$
\begin{aligned}
\Phi(\zeta, \eta)= & M^{\top}(\zeta) \Sigma M(\eta), \quad M \in \mathbb{R}^{\bullet \times \mathrm{w}}[\xi], \\
& \Sigma=\operatorname{diag}\left(I_{\mathrm{r}_{+}},-I_{\mathrm{r}_{-}}\right) \in \mathbb{R}_{\mathrm{s}}^{\bullet \times} \bullet
\end{aligned}
$$

Note that a constant matrix in the form of $\operatorname{diag}\left(I_{\mathrm{r}_{+}},-I_{\mathrm{r}_{-}}\right)$ is called a signature matrix. If $M(\xi)$ has minimal row number among such factorizations of $\Phi(\zeta, \eta)$, then we say that the factorization is a symmetric canonical factorization or simply a canonical factorization. Of course, the canonical factorization of a two-variable polynomial matrix is not unique. If we obtain two different canonical factorizations

$$
\Phi(\zeta, \eta)=M_{1}^{\top}(\zeta) \Sigma_{1} M_{1}(\eta)=M_{2}^{\top}(\zeta) \Sigma_{2} M_{2}(\eta),
$$

then there exists a nonsingular matrix $U \in \mathbb{R}^{\mathrm{v} \times \mathrm{v}}$ such that

$$
\Sigma_{2}=U^{\top} \Sigma_{1} U, \quad M_{1}(\xi)=U M_{2}(\xi) .
$$

Therefore, the canonical factorization is unique modulo a pseudo-unitary transformation $U$ (p.1709 in Willems and Trentelman 1998).

One of the important properties of QDF's is the fact that the derivative of a QDF is also a QDF. Namely, there holds

$$
\begin{aligned}
& \frac{d}{d t} Q_{\Phi}(w)(t)=Q_{\Psi}(w)(t) \quad \forall t \in \mathbb{R}, \forall w \in \mathfrak{C}^{\infty}\left(\mathbb{R}, \mathbb{R}^{\mathrm{w}}\right) \\
& \Leftrightarrow(\zeta+\eta) \Phi(\zeta, \eta)=\Psi(\zeta, \eta) .
\end{aligned}
$$

We hereafter use the notation

$$
\dot{\Phi}(\zeta, \eta):=(\zeta+\eta) \Phi(\zeta, \eta)
$$

Definition 1. Let $\Phi \in \mathbb{R}_{\mathrm{s}}^{\mathrm{w} \times \mathrm{w}}[\zeta, \eta]$ be given. $\mathrm{A} \operatorname{QDF} Q_{\Phi}$, or $\Phi(\zeta, \eta)$, is said to be nonnegative, denoted by $\Phi \geq 0$, if

$$
Q_{\Phi}(w)(t) \geq 0 \quad \forall t \in \mathbb{R}, \quad \forall w \in \mathfrak{C}^{\infty}\left(\mathbb{R}, \mathbb{R}^{\mathrm{w}}\right) .
$$

Furthermore, $Q_{\Phi}$, or $\Phi(\zeta, \eta)$, is called positive, denoted by $\Phi>0$, if it is nonnegative and $Q_{\Phi}(w)(t)=0 \forall t$ implies $w(t)=0 \forall t$. The nonpositivity and negativity of a QDF are also defined in the same way.
Proposition 1. Let $\Phi \in \mathbb{R}_{\mathrm{s}}^{\mathrm{w} \times \mathrm{w}}[\zeta, \eta]$ be given.

(i) The following are equivalent.

$$
\begin{aligned}
& \text { (a) } \Phi \geq 0 \text { (nonnegative). } \\
& \text { (b) There exists } D \in \mathbb{R}^{\bullet \times w}[\xi] \text { such that }
\end{aligned}
$$$$
\Phi(\zeta, \eta)=D^{\top}(\zeta) D(\eta) .
$$

(ii) The following are equivalent.

(a) $\Phi>0$ (positive).

(b) There exists $D \in \mathbb{R}^{\bullet \times w}[\xi]$ such that (1) holds, and $D(\lambda)$ has full column rank for all $\lambda \in \mathbb{C}$.

We often need to consider integrals of QDF's, in several applications such as LQ optimal control, $\mathcal{H}^{\infty}$ control, e.t.c. In particular, we are interested in boundedness or nonnegativity of such integrals.

Definition 2. Let $\Phi \in \mathbb{R}_{\mathrm{s}}^{\mathrm{w} \times \mathrm{w}}[\zeta, \eta]$ be given.

(i) A QDF $Q_{\Phi}$, or $\Phi(\zeta, \eta)$, is said to be average nonnegative, denoted by $\int Q_{\Phi} \geq 0$, if

$$
\int_{-\infty}^{\infty} Q_{\Phi}(w)(t) d t \geq 0 \quad \forall w \in \mathfrak{C}^{\infty}\left(\mathbb{R}, \mathbb{R}^{\mathrm{W}}\right) \cap \mathfrak{D} .
$$

(ii) $\mathrm{A} \mathrm{QDF} Q_{\Phi}$, or $\Phi(\zeta, \eta)$, is said to be half-line nonnegative, denoted by $\int^{t} Q_{\Phi} \geq 0$, if

$$
\int_{-\infty}^{0} Q_{\Phi}(w)(t) d t \geq 0 \quad \forall w \in \mathfrak{C}^{\infty}\left(\mathbb{R}, \mathbb{R}^{\mathrm{w}}\right) \cap \mathfrak{D} .
$$

The next proposition characterizes necessary and sufficient conditions for the average nonnegativity of $Q_{\Phi}$.

Proposition 2. The following are equivalent for $\Phi \in$ $\mathbb{R}_{\mathrm{s}}^{\mathrm{w} \times \mathrm{w}}[\zeta, \eta]$.

(i) $\int Q_{\Phi} \geq 0$ (average nonnegative).

(ii) $\Phi(\bar{\lambda}, \lambda) \geq 0 \quad \forall \lambda \in \mathrm{i} \mathbb{R}$

(iii) There exists $\Psi \in \mathbb{R}_{\mathrm{S}}^{\mathrm{w} \times \mathrm{w}}[\zeta, \eta]$ such that

$$
\frac{d}{d t} Q_{\Psi}(w)(t) \leq Q_{\Phi}(w)(t) \forall t \in \mathbb{R}, \forall w \in \mathfrak{C}^{\infty}\left(\mathbb{R}, \mathbb{R}^{\mathrm{W}}\right) .
$$

(iv) There exist $\Psi \in \mathbb{R}_{\mathrm{s}}^{\mathrm{w} \times \mathrm{w}}[\zeta, \eta]$ and $F \in \mathbb{R}^{\bullet \times \mathrm{w}}[\xi]$ such that

$$
(\zeta+\eta) \Psi(\zeta, \eta)+F^{\top}(\zeta) F(\eta)=\Phi(\zeta, \eta)
$$

The inequality (4) is closely related to the dissipativity of a linear dynamical system. In fact, the inequality (4) is referred to as a dissipation inequality, in which $Q_{\Phi}$ and $Q_{\Psi}$ are called a supply rate and a storage function, respectively. The reason for these names is explained as follows.

Let a linear dynamical system $\mathfrak{S}$ be described by the image representation $v=M\left(\frac{d}{d t}\right) w, M \in \mathbb{R}^{\mathrm{v} \times \mathrm{w}}[\xi]$, where $w$ is the free latent variable, and $v$ the manifest variable which describes the external behavior of the system. Consider the quadratic form $s(t)=v^{\top}(t) \Sigma v(t), \Sigma \in \mathbb{R}_{\mathrm{s}}^{\mathrm{v} \times \mathrm{v}}$. Using the image representation, $s(t)$ can be expressed as a QDF of the latent variable $w$ as

$$
s=Q_{\Phi}(w)=\left(M\left(\frac{d}{d t}\right) w\right)^{\top} \Sigma\left(M\left(\frac{d}{d t}\right) w\right),
$$

where $\Phi(\zeta, \eta)=M^{\top}(\zeta) \Sigma M(\eta)$. If we view $s(t)=$ $Q_{\Phi}(w)(t)$ as the rate of energy supply into the system $\mathfrak{S}$ and $Q_{\Psi}(w)(t)$ as the energy stored in $\mathfrak{S}$, then (4) claims that the rate of increase of the stored energy $Q_{\Psi}(w)$ never exceeds the rate of energy supply $Q_{\Phi}$, namely, the system $\mathfrak{S}$ dissipates energy. 
If $Q_{\Phi}$ is average nonnegative, a storage function can be obtained by the following procedure. First, we perform the polynomial spectral factorization

$$
\Phi(-\xi, \xi)=F^{\top}(-\xi) F(\xi) .
$$

This factorization is possible whenever the condition (ii) in the above proposition is satisfied. Then, one of the storage functions for $Q_{\Phi}$ is induced by

$$
\Psi(\zeta, \eta)=\frac{\Phi(\zeta, \eta)-F^{\top}(\zeta) F(\eta)}{\zeta+\eta}
$$

Clearly, the resulting $\Psi(\zeta, \eta)$ and $F(\xi)$ satisfy (5).

It is seen from (5) that there holds

$$
\begin{aligned}
\frac{d}{d t} Q_{\Psi}(w)(t)+Q_{\Delta}(w)(t) & =Q_{\Phi}(w)(t) \\
\forall t & \in \mathbb{R}, \quad \forall w \in \mathfrak{C}^{\infty}\left(\mathbb{R}, \mathbb{R}^{\mathrm{w}}\right),
\end{aligned}
$$

where we have defined $\Delta(\zeta, \eta)=F^{\top}(\zeta) F(\eta)$, and $Q_{\Delta}$ is called a dissipation rate. Note that $\Delta \geq 0$ holds by its definition and Proposition 1 (i). It is also immediate from (8) that

$\int_{-\infty}^{\infty} Q_{\Delta}(w)(t) d t=\int_{-\infty}^{\infty} Q_{\Phi}(w)(t) d t \forall w \in \mathfrak{C}^{\infty}\left(\mathbb{R}, \mathbb{R}^{\mathrm{w}}\right) \cap \mathfrak{D}$.

Hence, as another characterization of the average nonnegativity (dissipativity) of $Q_{\Phi}$, we conclude that $\int Q_{\Phi} \geq 0$ is equivalent to the existence of a dissipation rate $Q_{\Delta}$ satisfying $\Delta \geq 0$ and (9).

A nonnegative storage function often plays an important role in many issues arising in system and control theory such as stability analyses.

The next proposition relates the half-line positivity in Definition 2 (ii) and the existence of a nonnegative storage function.

Proposition 3. Let $\Phi \in \mathbb{R}_{\mathrm{s}}^{\mathrm{w} \times \mathrm{w}}[\zeta, \eta]$ be given. The following are equivalent.

(i) $Q_{\Phi}$ is average nonnegative and admits a nonnegative storage function.

(ii) $\int^{t} Q_{\Phi} \geq 0$ (half-line nonnegative).

Remark 1. A frequency domain condition for the half-line nonnegativity of $Q_{\Phi}$ is given in terms of a so-called Pick matrix. For the detail, interested readers are recommended to refer to Section 9 in Willems and Trentelman (1998).

\section{DIFFERENTIAL EQUATION REPRESENTED BY RATIONAL FUNCTIONS}

Before generalizing the polynomial QDF in the previous section to a rational QDF (its precise definition will be presented in the next section), we consider a linear differential equation represented by

$$
v=G\left(\frac{d}{d t}\right) w, \quad G \in \mathbb{R}^{\mathrm{v} \times \mathrm{w}}(\xi) .
$$

For more various rational function representations such as $G\left(\frac{d}{d t}\right) w=0$ and $G\left(\frac{d}{d t}\right) w=H\left(\frac{d}{d t}\right) \ell$, the readers are recommended to refer to Willems and Yamamoto (2007).

We introduce left and right coprime factorizations of $G(\xi)$ over $\mathbb{R}[\xi]$ as

$$
\begin{gathered}
G(\xi)=X^{-1}(\xi) Y(\xi)=N(\xi) D^{-1}(\xi), \\
X \in \mathbb{R}^{\mathrm{v} \times \mathrm{v}}[\xi], \quad Y \in \mathbb{R}^{\mathrm{v} \times \mathrm{w}}[\xi], \\
N \in \mathbb{R}^{\mathrm{v} \times \mathrm{w}}[\xi], \quad D \in \mathbb{R}^{\mathrm{w} \times \mathrm{w}}[\xi] .
\end{gathered}
$$

Then, along the line of Willems and Yamamoto (2007), the solution of (10) is defined as follows.

Definition 3. The pair $(v, w) \in \mathfrak{C}^{\infty}\left(\mathbb{R}, \mathbb{R}^{\mathrm{v}+\mathrm{w}}\right)$ is a solution of (11) if there holds

$$
X\left(\frac{d}{d t}\right) v=Y\left(\frac{d}{d t}\right) w .
$$

Proposition 4. Let $G \in \mathbb{R}^{\mathrm{v} \times \mathrm{w}}(\xi)$ be given. Then, $(v, w) \in$ $\mathfrak{C}^{\infty}\left(\mathbb{R}, \mathbb{R}^{\mathrm{v}+\mathrm{w}}\right)$ is a solution of (10) if and only if there exists $\ell \in \mathfrak{C}^{\infty}\left(\mathbb{R}, \mathbb{R}^{\mathrm{w}}\right)$ such that

$$
\left(\begin{array}{c}
v \\
w
\end{array}\right)=\left(\begin{array}{l}
N\left(\frac{d}{d t}\right) \\
D\left(\frac{d}{d t}\right)
\end{array}\right) \ell
$$

Proof: The proof is straightforward from the definitions of coprime factors in (11) and the relation between kernel and image representations (see e.g. Section 6.6 in Polderman and Willems (1998)).

Proposition 5. Consider a solution to the differential equation (13) associated with the rational representation $v=$ $G\left(\frac{d}{d t}\right) w$.

(i) $w \in \mathfrak{C}^{\infty}\left(\mathbb{R}, \mathbb{R}^{w}\right) \Leftrightarrow \ell \in \mathfrak{C}^{\infty}\left(\mathbb{R}, \mathbb{R}^{w}\right)$.

(ii) $(v, w) \in \mathfrak{C}^{\infty}\left(\mathbb{R}, \mathbb{R}^{\mathrm{v}+\mathrm{w}}\right)$ has compact support iff $\ell \in$ $\mathfrak{C}^{\infty}\left(\mathbb{R}, \mathbb{R}^{\mathrm{w}}\right)$ has compact support.

Proof: (i) Immediate.

(ii) By the right coprimeness, $\left(\begin{array}{l}N(\lambda) \\ D(\lambda)\end{array}\right)$ has full column rank for all $\lambda \in \mathbb{C}$. Hence, $\ell$ is observable from $(v, w)$, namely $(v, w)=(0,0)$ implies $\ell=0$. This proves the statement in (ii).

Consider the situation where $w$ is arbitrarily given and $v$ is determined from $v=G\left(\frac{d}{d t}\right) w$ (equivalently (12)). In general, $G\left(\frac{d}{d t}\right)$ does not define a point-to-point map from $w$ to $v$, but a point-to-set map. This is because there are a number of $v$ 's satisfying (10). In fact, given a solution $(v, w)$ of $(10),\left(v+v^{\prime}, w\right)$ is also a solution of (10) for any $v^{\prime}$ satisfying $X\left(\frac{d}{d t}\right) v^{\prime}=0$. With abuse of notation, we define

$$
G\left(\frac{d}{d t}\right) w:=\left\{v \in \mathfrak{C}^{\infty}\left(\mathbb{R}, \mathbb{R}^{\mathrm{v}}\right) \mid X\left(\frac{d}{d t}\right) v=Y\left(\frac{d}{d t}\right) w\right\}
$$

for a given $w \in \mathfrak{C}^{\infty}\left(\mathbb{R}, \mathbb{R}^{\mathrm{w}}\right)$, so that $v=G\left(\frac{d}{d t}\right) w$ is equivalent to $v \in G\left(\frac{d}{d t}\right) w$. Of course, in the case where $G(\xi)$ is a polynomial matrix, $G\left(\frac{d}{d t}\right)$ defines a point-topoint map, and hence $G\left(\frac{d}{d t}\right) w$ is a singleton for any $w \in$ $\mathfrak{C}^{\infty}\left(\mathbb{R}, \mathbb{R}^{\mathrm{w}}\right)$.

It should be noted that Propositions 4 and 5 claim that

$$
\mathfrak{B}=\left\{v \in \mathfrak{C}^{\infty}\left(\mathbb{R}, \mathbb{R}^{\mathrm{v}}\right) \mid \exists w \text { s.t. } v \in G\left(\frac{d}{d t}\right) w\right\}
$$

can be represented as the image space of the differential operator $N\left(\frac{d}{d t}\right)$, namely

$$
\mathfrak{B}=\left\{v \in \mathfrak{C}^{\infty}\left(\mathbb{R}, \mathbb{R}^{\mathrm{v}}\right) \mid \exists \ell \text { s.t. } v=N\left(\frac{d}{d t}\right) \ell\right\} .
$$

\section{RATIONAL QUADRATIC DIFFERENTIAL FORMS}

The rest of this paper is devoted to more general QDF's defined by rational functions, which we call rational $Q D F$ 's. 
Quadratic constraints on rational transfer functions such as bounded realness, positive realness, integral quadratic constraints (IQC's) and quadratic separators (see e.g. Megretzki and Rantzer 1997, Iwasaki and Hara 1998) have been playing a crucial role in the analyses of linear dynamical systems (especially, stability analysis of feedback systems). Also, by using state-space representations, the well-known Kalman-Yakubovich-Popov lemma clarifies a connection between such constraints and dissipativity.

The purpose of this section is to introduce the notion of rational QDF's as the foundation for studying the above quadratic constraints on rational functions from viewpoint of the behavioral approach.

\subsection{Definition}

We now formulate a rational QDF induced by a symmetric two-variable rational matrix $\Phi \in \mathbb{R}_{s}^{\mathrm{w} \times \mathrm{w}}(\zeta, \eta)$ rather than a two-variable polynomial matrix.

Definition 4. A symmetric two-variable rational matrix $\Phi \in \mathbb{R}_{\mathrm{s}}^{\mathrm{W} \times \mathrm{w}}(\zeta, \eta)$ is said to be factorizable if there exist a rational matrix $G \in \mathbb{R}^{\bullet \times w}(\xi)$ and a signature matrix $\Sigma \in \mathbb{R}_{\mathrm{S}}^{\bullet} \bullet$ such that

$$
\Phi(\zeta, \eta)=G^{\top}(\zeta) \Sigma G(\eta)
$$

It is straightforward to show that $\Phi(\zeta, \eta)$ is factorizable iff the least common multiple of the denominators of all entries of $\Phi(\zeta, \eta)$ is factored as $\varphi(\zeta) \varphi(\eta)$, namely, $\Phi(\zeta, \eta)$ is expressed as

$$
\Phi(\zeta, \eta)=\frac{\Pi(\zeta, \eta)}{\varphi(\zeta) \varphi(\eta)}, \quad \Pi \in \mathbb{R}_{\mathrm{s}}^{\mathrm{w} \times \mathrm{w}}[\zeta, \eta], \quad \varphi \in \mathbb{R}[\xi] .
$$

Of course, the factorization in (15) is not unique. We say that the factorization $\Phi(\zeta, \eta)=G^{\top}(\zeta) \Sigma G(\eta)$ is canonical, if $\operatorname{row}(G) \leq \operatorname{row}\left(G^{\prime}\right)$ for any other $G^{\prime} \in \mathbb{R}^{\bullet \times w}(\xi)$ and $\Sigma^{\prime}=\operatorname{diag}\left(\bar{I}_{\mathrm{r}_{+}},-I_{\mathrm{r}_{-}}\right) \in \mathbb{R}_{\mathrm{s}}^{\bullet} \bullet$ satisfying $\Phi(\zeta, \eta)=$ $G^{\prime \top}(\zeta) \Sigma^{\prime} G^{\prime}(\eta)$, where row $(\cdot)$ denotes the number of rows of a (rational) matrix.

We hereafter make the following assumption.

Assumption 1. The two-variable rational matrix $\Phi \in$ $\mathbb{R}_{\mathrm{S}}^{\mathrm{W} \times \mathrm{w}}(\zeta, \eta)$ is symmetric, factorizable and admits a canonical factorization in the form of $(15)$ with $\mathrm{v}:=\operatorname{row}(G)$.

We now at the position to define a rational QDF.

Definition 5. Under Assumption 1, the rational QDF with respect to $\Phi$ is the set defined by

$Q_{\Phi}^{\mathbf{r}}(w):=\left\{s \in \mathfrak{C}^{\infty}(\mathbb{R}, \mathbb{R}) \mid \exists v \in G\left(\frac{d}{d t}\right) w\right.$ s.t. $\left.s=v^{\top} \Sigma v\right\}$.

Note that we put the superscript " $\mathbf{r}$ " in order to distinguish the rational QDF from polynomial QDF's.

The rational QDF is well-defined in the sense that it is uniquely defined regardless of the choice of the canonical factorization. The reason of this uniqueness is as follows. Suppose that $\Phi \in \mathbb{R}_{\mathrm{s}}^{\mathrm{w} \times \mathrm{w}}(\zeta, \eta)$ is expressed as in (16). Moreover, assume that $\Phi(\zeta, \eta)$ admits two different canonical factorizations as

$$
\Phi(\zeta, \eta)=G_{1}^{\top}(\zeta) \Sigma_{1} G_{1}(\eta)=G_{2}^{\top}(\zeta) \Sigma_{2} G_{2}(\eta)
$$

By the definition of the canonical factorization, we must have $\operatorname{row}\left(G_{1}\right)=\operatorname{row}\left(G_{2}\right)=$ : v. Then, it is obvious that $\varphi(\xi) G_{i}(\xi)(i=1,2)$ is a polynomial matrix in $\mathbb{R}^{\mathrm{v} \times \mathrm{w}}[\xi]$ and induces a canonical factorization of $\Pi(\zeta, \eta)$ in $(16)$ in the polynomial matrix sense. (Otherwise, it can be shown that $G_{i}(\xi)$ is not a canonical factor of $\Phi(\zeta, \eta)$.) As discussed in Section 2, there exists a nonsingular constant matrix $U$ such that $\varphi(\xi) G_{1}(\xi)=U \varphi(\xi) G_{2}(\xi)$ and $U^{\top} \Sigma_{1} U=\Sigma_{2}$. Hence, we have $G_{1}(\xi)=U G_{2}(\xi)$. As a result, we see that the rational QDF's induced from two different canonical factors are identical, because there holds

$$
\begin{aligned}
& \left\{s \in \mathfrak{C}^{\infty}(\mathbb{R}, \mathbb{R}) \mid \exists v_{1} \in G_{1}\left(\frac{d}{d t}\right) w \text { s.t. } s=v_{1}^{\top} \Sigma_{1} v_{1}\right\} \\
& =\left\{s \in \mathfrak{C}^{\infty}(\mathbb{R}, \mathbb{R}) \mid \exists v_{1} \in U G_{2}\left(\frac{d}{d t}\right) w \text { s.t. } s=v_{1}^{\top} \Sigma_{1} v_{1}\right\} \\
& =\left\{s \in \mathfrak{C}^{\infty}(\mathbb{R}, \mathbb{R}) \mid \exists v_{2} \in G_{2}\left(\frac{d}{d t}\right) w \text { s.t. } s=v_{2}^{\top} U^{\top} \Sigma_{1} U v_{2}\right\} \\
& =\left\{s \in \mathfrak{C}^{\infty}(\mathbb{R}, \mathbb{R}) \mid \exists v_{2} \in G_{2}\left(\frac{d}{d t}\right) w \text { s.t. } s=v_{2}^{\top} \Sigma_{2} v_{2}\right\},
\end{aligned}
$$

where the most left- and right-hand sides are the rational QDF's induced by $G_{1}(\xi)$ and $G_{2}(\xi)$, respectively.

\section{Example 1.}

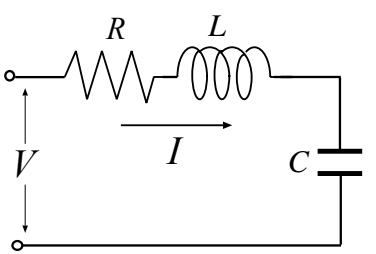

Fig. 1. RLC electrical network

We consider a simple electrical network consisting of a resistor $R$, an inductor $L$ and a capacitor $C$ (Fig. 1). Let $V(t)$ and $I(t)$ be the port voltage and current at time $t$, respectively. We also define $q(t)$ as the electric charge in the capacitor. Using fundamental laws of electrical elements and circuits, the dynamics of this system is described by

$$
\frac{d V}{d t}=\frac{1}{C} I+R \frac{d I}{d t}+L \frac{d^{2} I}{d t^{2}}
$$

Then, a rational representation of this system is given by

$$
\left(\begin{array}{c}
V \\
I
\end{array}\right)=\left(\begin{array}{c}
1 \\
H\left(\frac{d}{d t}\right)
\end{array}\right) V, \quad H(\xi)=\frac{\xi}{\frac{1}{C}+R \xi+L \xi^{2}} .
$$

As well known, the instantaneous power $s(t)$ into the electrical network is the product of the voltage and the current, which is expressed in the quadratic form as

$$
s=V I=\left(\begin{array}{ll}
V & I
\end{array}\right)\left(\begin{array}{cc}
0 & 1 / 2 \\
1 / 2 & 0
\end{array}\right)\left(\begin{array}{l}
V \\
I
\end{array}\right) .
$$

In view of (18), this is associated with the rational matrix $\Phi(\zeta, \eta)=\frac{1}{2}(1 H(\zeta))\left(\begin{array}{ll}0 & 1 \\ 1 & 0\end{array}\right)\left(\begin{array}{c}1 \\ H(\eta)\end{array}\right)=\frac{1}{2}(H(\zeta)+H(\eta))$.

One of the canonical factorizations of $\Phi(\zeta, \eta)$ is given by

$$
G(\xi)=\frac{1}{2}\left(\begin{array}{c}
1+H(\xi) \\
1-H(\xi)
\end{array}\right), \quad \Sigma=\left(\begin{array}{cc}
1 & 0 \\
0 & -1
\end{array}\right)
$$

Therefore, the power $s$ can be expressed as the rational QDF with

$s=v^{\top}\left(\begin{array}{cc}1 & 0 \\ 0 & -1\end{array}\right) v, w=V, v=G\left(\frac{d}{d t}\right) V=\frac{1}{2}\left(\begin{array}{c}V+I \\ V-I\end{array}\right)$. 
Proposition 6. For two symmetric rational matrices $\Phi_{1}$, $\Phi_{2} \in \mathbb{R}_{\mathrm{s}}^{\mathrm{W} \times \mathrm{w}}(\zeta, \eta)$, we have

$$
Q_{\Phi_{1}+\Phi_{2}}^{\mathbf{r}}(w) \subset Q_{\Phi_{1}}^{\mathbf{r}}(w)+Q_{\Phi_{2}}^{\mathbf{r}}(w) \quad \forall w \in \mathfrak{C}^{\infty}\left(\mathbb{R}, \mathbb{R}^{\mathrm{w}}\right) .
$$

Proof. Straightforward from Definition 5 and the definition of the canonical factorization.

Let the right coprime factorization of $G(\xi)$ in (15) over $\mathbb{R}[\xi]$ be given by

$$
G(\xi)=N(\xi) D^{-1}(\xi), \quad N \in \mathbb{R}^{\mathrm{v} \times \mathrm{w}}[\xi], D \in \mathbb{R}^{\mathrm{w} \times \mathrm{w}}[\xi] .
$$

Then, we see from Section 3 that the pair $(v, w)$ satisfying $v=G\left(\frac{d}{d t}\right) w$ belongs to $\mathfrak{C}^{\infty}\left(\mathbb{R}, \mathbb{R}^{\mathrm{v}+\mathrm{w}}\right)$ iff there exists $\ell \in$ $\mathfrak{C}^{\infty}\left(\mathbb{R}, \mathbb{R}^{\mathrm{w}}\right)$ satisfying (13). Since $s=v^{\top} \Sigma v$ is rewritten as $s=\left(N\left(\frac{d}{d t}\right) \ell\right)^{\top} \Sigma\left(N\left(\frac{d}{d t}\right) \ell\right)=Q_{N^{\top}(\zeta) \Sigma N(\eta)}(\ell)$, we have

$$
Q_{\Phi}^{\mathbf{r}}(w)=\left\{Q_{N^{\top}(\zeta) \Sigma N(\eta)}(\ell) \mid \quad \ell \in D^{-1}\left(\frac{d}{d t}\right) w\right\} .
$$

Therefore, the rational QDF $Q_{\Phi}^{\mathrm{r}}$ inherits the properties (e.g. nonnegativity, average nonnegativity, e.t.c.) of the polynomial QDF $Q_{N^{\top}(\zeta) \Sigma N(\eta)}$ as discussed below.

In the same way as the polynomial case, we introduce the notation

$$
\dot{\Phi}(\zeta, \eta)=(\zeta+\eta) \Phi(\zeta, \eta)
$$

Proposition 7. Let a symmetric rational matrix $\Phi \in$ $\mathbb{R}_{\mathrm{S}}^{\mathrm{w} \times \mathrm{w}}(\zeta, \eta)$ be given. Then, under Assumption 1 , we obtain

$$
Q_{\dot{\Phi}}^{\mathbf{r}}(w)=\left\{r \in \mathfrak{C}^{\infty}(\mathbb{R}, \mathbb{R}) \mid \exists s \in Q_{\Phi}^{\mathbf{r}}(w) \text { s.t. } r=\frac{d}{d t} s\right\} .
$$

Proof: Let a right coprime factorization be given by (20). We also define $\Theta(\zeta, \eta)$ by

$$
\Theta(\zeta, \eta):=D^{\top}(\zeta) \Phi(\zeta, \eta) D(\eta)=N^{\top}(\zeta) \Sigma N(\eta)
$$

Then, $\Theta(\zeta, \eta)$ is a symmetric polynomial matrix in $\mathbb{R}^{\mathrm{w} \times \mathrm{w}}[\zeta, \eta]$. Moreover, let $M \in \mathbb{R}^{\mathrm{v} \times \mathrm{w}}[\xi]$ and $\Sigma^{\prime} \in \mathbb{R}_{\mathrm{s}}^{\mathrm{v} \times \mathrm{v}}$ induce a canonical factorization

Then, there holds

$$
(\zeta+\eta) \Theta(\zeta, \eta)=M^{\top}(\zeta) \Sigma^{\prime} M(\eta)
$$

$$
\begin{aligned}
\frac{d}{d t}\left\{\left(N\left(\frac{d}{d t}\right) \ell\right)^{\top} \Sigma\left(N\left(\frac{d}{d t}\right) \ell\right)\right\}= & \left(M\left(\frac{d}{d t}\right) \ell\right)^{\top} \Sigma^{\prime}\left(M\left(\frac{d}{d t}\right) \ell\right) \\
& \forall \ell \in \mathfrak{C}^{\infty}\left(\mathbb{R}, \mathbb{R}^{\mathrm{w}}\right) .
\end{aligned}
$$

By the definition of the canonical factorization, it can be shown that the pair $(M, D)$ is right coprime. We also see that $H(\xi):=M(\xi) D^{-1}(\xi)$ is a canonical factor of $\dot{\Phi}(\zeta, \eta)$. As a result, we get

$$
\begin{aligned}
Q_{\dot{\Phi}}^{\mathbf{r}}(w) & =\left\{r \in \mathfrak{C}^{\infty}(\mathbb{R}, \mathbb{R}) \mid \exists z \in H\left(\frac{d}{d t}\right) w \text { s.t. } r=z^{\top} \Sigma^{\prime} z\right\} \\
& =\left\{r=z^{\top} \Sigma^{\prime} z \mid z=M\left(\frac{d}{d t}\right) \ell \& \ell \in D^{-1}\left(\frac{d}{d t}\right) w\right\} \\
& =\left\{Q_{M^{\top}(\zeta) \Sigma^{\prime} M(\eta)}(\ell) \mid \ell \in D^{-1}\left(\frac{d}{d t}\right) w\right\} \\
& =\left\{\frac{d}{d t} Q_{N^{\top}(\zeta) \Sigma^{\prime} N(\eta)}(\ell) \mid \ell \in D^{-1}\left(\frac{d}{d t}\right) w\right\} \\
& =\left\{\frac{d}{d t} s \mid \exists \ell \in D^{-1}\left(\frac{d}{d t}\right) w \text { s.t. } s=Q_{N^{\top}(\zeta) \Sigma^{\prime} N(\eta)}(\ell)\right\} \\
& =\left\{\frac{d}{d t} s \mid s \in Q_{\Phi}^{\mathbf{r}}(w)\right\} .
\end{aligned}
$$

This completes the proof.

\subsection{Nonnegativity of rational $Q D F$}

Definition 6. Under Assumption 1, $Q_{\Phi}^{\mathrm{r}}$ is said to be nonnegative, denoted by $\Phi \geq 0$, if

$$
\left.\llbracket\left[s(t) \geq 0 \forall t \in \mathbb{R} \forall s \in Q_{\Phi}^{\mathbf{r}}(w)\right]\right] \forall w \in \mathfrak{C}^{\infty}\left(\mathbb{R}, \mathbb{R}^{\mathrm{w}}\right) .
$$

The nonpositivity of $Q_{\Phi}^{\mathbf{r}}$ is also defined in the same way.
Proposition 8. Under Assumption 1,

$$
\Phi \geq 0 \Leftrightarrow \exists K \in \mathbb{R}^{\bullet \times w}(\xi) \text { s.t. } \Phi(\zeta, \eta)=K^{\top}(\zeta) K(\eta) \text {. }
$$

\section{Proof:}

$(\Leftarrow)$ Obvious because we can assume without loss of generality that $\Phi(\zeta, \eta)=K^{\top}(\zeta) K(\eta)$ is a canonical factorization. Even if this is not the case, we can easily show that the canonical factorization is given in the form of $\Phi(\zeta, \eta)=G^{\top}(\zeta) G(\eta), \Sigma=I_{\mathrm{v}}$.

$(\Rightarrow)$ Under Assumption 1, we again introduce a right coprime factorization of (20). It then follows from (21) and Definition 6 that

$$
\begin{aligned}
\Phi \geq 0 \Leftrightarrow \llbracket s(t)= & Q_{N^{\top}(\zeta) \Sigma N(\eta)}(\ell)(t) \geq 0 \forall t \in \mathbb{R}, \\
& \forall \ell \in D^{-1}\left(\frac{d}{d t}\right) w, \forall w \in \mathfrak{C}^{\infty}\left(\mathbb{R}, \mathbb{R}^{\mathrm{w}}\right) \rrbracket .
\end{aligned}
$$

As shown in Proposition $5(\mathrm{i})$, we see that $w \in \mathfrak{C}^{\infty}\left(\mathbb{R}, \mathbb{R}^{\mathrm{w}}\right) \Leftrightarrow$ $\ell \in \mathfrak{C}^{\infty}\left(\mathbb{R}, \mathbb{R}^{\mathrm{w}}\right)$ for any solution of $w=D\left(\frac{d}{d t}\right) \ell$. Therefore, we have

$$
\begin{gathered}
\Phi \geq 0 \Leftrightarrow Q_{N^{\top}(\zeta) \Sigma N(\eta)}(\ell)(t) \geq 0 \forall t \in \mathbb{R}, \forall \ell \in \mathfrak{C}^{\infty}\left(\mathbb{R}, \mathbb{R}^{\mathrm{w}}\right) \\
\left.\Leftrightarrow N^{\top}(\zeta) \Sigma N(\eta) \geq 0 \text { (in the sense of polynomial } Q D F^{\prime} s\right) \\
\Leftrightarrow \exists F \in \mathbb{R}^{\bullet \times \mathrm{w}}[\xi] \text { s.t. } N^{\top}(\zeta) \Sigma N(\eta)=F^{\top}(\zeta) F(\eta) \\
\text { (by Proposition } 1(\mathrm{i}) \text { ) }
\end{gathered}
$$

As a result, by defining $K(\xi)=F(\xi) D^{-1}(\xi)$, we obtain $\Phi(\zeta, \eta)=K^{\top}(\zeta) K(\eta)$. This completes the proof.

Also, the inequality between two rational QDF's is defined as follows.

$\Phi_{1}-\Phi_{2} \geq 0$

$: \Leftrightarrow \llbracket s(t) \geq 0 \forall t \in \mathbb{R}, \forall s \in Q_{\Phi_{1}-\Phi_{2}}^{\mathbf{r}}(w), \forall w \in \mathfrak{C}^{\infty}\left(\mathbb{R}, \mathbb{R}^{\mathrm{w}}\right) \rrbracket$ Note that $\Phi_{1}-\Phi_{2} \geq 0$ does not imply $s_{1}(t) \geq s_{2}(t) \forall t \in \mathbb{R}$, $\forall s_{i} \in Q_{\Phi_{i}}^{\mathbf{r}}(w)(i=1,2), \forall w \in \mathfrak{C}^{\infty}\left(\mathbb{R}, \mathbb{R}^{\mathrm{w}}\right)$.

\subsection{Average nonnegativity and dissipation inequality}

In order to define the average nonnegativity, we introduce the set $\hat{Q}_{\Phi}^{\mathrm{r}}(w)$ as

$\hat{Q}_{\Phi}^{\mathrm{r}}(w)=\left\{s \in \mathfrak{C}^{\infty}(\mathbb{R}, \mathbb{R}) \mid \exists v \in G\left(\frac{d}{d t}\right) w \cap \mathfrak{D}\right.$ s.t. $\left.s=v^{\top} \Sigma v\right\}$. Clearly, $\hat{Q}_{\Phi}^{\mathbf{r}}(w)$ is a subset of $Q_{\Phi}^{\mathbf{r}}(w)$, and any $s \in \hat{Q}_{\Phi}^{\mathbf{r}}(w)$ has compact support, guaranteeing the existence of the integral $\int_{-\infty}^{\infty} s(t) d t$.

Definition 7. Under Assumption 1, $Q_{\Phi}^{\mathbf{r}}$ is called average nonnegative, denoted by $\int Q_{\Phi}^{\mathbf{r}} \geq 0$, if there holds

$$
\int_{-\infty}^{\infty} s(t) d t \geq 0 \quad \forall s \in \hat{Q}_{\Phi}^{\mathrm{r}}(w), \forall w \in \mathfrak{C}^{\infty}\left(\mathbb{R}, \mathbb{R}^{\mathrm{w}}\right) \cap \mathfrak{D} .
$$

The average nonpositivity is also defined in the same way.

It may be noted that the integral inequality above can be interpreted as a kind of integral quadratic constraints (Megretski and Rantzer 1997) in the time domain.

Lemma 1. Under Assumption $1, Q_{\Phi}^{\mathbf{r}}$ is average nonnegative if and only if there exist a factorizable $\Psi \in \mathbb{R}_{\mathrm{s}}^{\mathrm{w} \times \mathrm{w}}(\zeta, \eta)$ and an $F \in \mathbb{R}^{\bullet \times w}(\xi)$ such that

$$
(\zeta+\eta) \Phi(\zeta, \eta)+F^{\top}(\zeta) F(\eta)=\Phi(\zeta, \eta) .
$$

\section{Proof:}

$(\Rightarrow)$ In view of (13) and Proposition 5 (ii), $(v, w)$ has compact support iff so does $\ell$. It thus follows that 


$$
\begin{aligned}
& \int Q_{\Phi}^{\mathrm{r}} \geq 0 \\
& \Leftrightarrow \int_{-\infty}^{\infty} v^{\top}(t) \Sigma v(t) d t \geq 0 \quad \forall v \in G\left(\frac{d}{d t}\right) w \cap \mathfrak{D}, \\
& \quad \forall w \in \mathfrak{C}^{\infty}\left(\mathbb{R}, \mathbb{R}^{\mathrm{w}}\right) \cap \mathfrak{D}
\end{aligned}
$$

Pre- and post-multiplying (26) by $D^{-\top}(\zeta)$ and $D^{-1}(\eta)$, respectively, we obtain $(25)$ with

$$
\begin{aligned}
\Psi(\zeta, \eta) & :=D^{-\top}(\zeta) \hat{\Psi}(\zeta, \eta) D^{-1}(\eta), \\
F(\xi) & :=\hat{F}(\xi) D^{-1}(\xi) .
\end{aligned}
$$

It is clear from this definition that $\Psi(\zeta, \eta)$ is factorizable.

$(\Rightarrow)$ Since $\Phi(\zeta, \eta)=G^{\top}(\zeta) \Sigma G(\eta)$ and $G(\xi)=N(\xi) D^{-1}(\xi)$, it follows from (25) that

$$
\begin{array}{r}
(\zeta+\eta) D^{\top}(\zeta) \Psi(\zeta, \eta) D(\eta)+(F(\zeta) D(\zeta))^{\top} F(\eta) D(\eta) \\
=N^{\top}(\zeta) \Sigma N(\eta) .
\end{array}
$$

To prove the sufficiency, we first show that we can always find a solution of (25) such that both $D^{\top}(\zeta) \Psi(\zeta, \eta) D(\eta)$ and $F(\eta) D(\eta)$ are polynomial matrices. Suppose that this is not the case. It follows from (27) that

$$
N^{\top}(-\xi) \Sigma N(\xi)=(F(-\xi) D(-\xi))^{\top} F(\xi) D(\xi) .
$$

Since the left-hand side is a polynomial matrix, $F(\xi) D(\xi)$ must be factored as

$$
F(\xi) D(\xi)=U(\xi) W(\xi),
$$

where $W(\xi)$ is a polynomial matrix, and $U(\xi)$ is a unitary rational matrix, namely $U^{\top}(-\xi) U(\xi)=I$. Then, we redefine $F(\xi)$ and $\Psi(\zeta, \eta)$ as

$$
F(\xi) \leftarrow W(\xi) D^{-1}(\xi),
$$

$\Psi(\zeta, \eta) \leftarrow \Psi(\zeta, \eta)+\frac{1}{\zeta+\eta}\left\{F^{\top}(\zeta) F(\eta)-D^{-\top}(\zeta) W^{\top}(\zeta) W(\eta) D^{-1}(\eta)\right\}$

Note that this new $\Psi(\zeta, \eta)$ is still factorizable because $F^{\top}(\zeta) F(\eta)-D^{-\top}(\zeta) W^{\top}(\zeta) W(\eta) D^{-1}(\eta)$ has $\zeta+\eta$ as a factor of its numerator. Furthermore, since $(\zeta+$ $\eta) D^{\top}(\zeta) \Psi(\zeta, \eta) D(\eta)$ is a polynomial matrix from $(27)$, we see that $D^{\top}(\zeta) \Psi(\zeta, \eta) D(\eta)$ is a polynomial matrix. Thus, we hereafter assume that both $D^{\top}(\zeta) \Psi(\zeta, \eta) D(\eta)$ and $F(\eta) D(\eta)$ are polynomial matrices.

Next, we define

$$
\begin{aligned}
& \Xi(\zeta, \eta)=D^{\top}(\zeta) \Psi(\zeta, \eta) D(\eta), \\
& \Delta(\zeta, \eta)=(F(\zeta) D(\zeta))^{\top} F(\eta) D(\eta) .
\end{aligned}
$$

Since $\Xi(\zeta, \eta)$ and $\Delta(\zeta, \eta)$ are polynomial matrices, the polynomial QDF's $Q_{\Xi}$ and $Q_{\Delta}$ satisfy

$$
\begin{aligned}
Q_{N^{\top}(\zeta) \Sigma N(\eta)}(\ell)(t)= & \frac{d}{d t} Q_{\Xi}(\ell)(t)+Q_{\Delta}(\ell)(t) \\
& \forall t \in \mathbb{R}, \forall \ell \in \mathfrak{C}^{\infty}\left(\mathbb{R}, \mathbb{R}^{\mathrm{w}}\right) .
\end{aligned}
$$

Hence, for every $\ell \in \mathfrak{C}^{\infty}\left(\mathbb{R}, \mathbb{R}^{\mathrm{w}}\right) \cap \mathfrak{D}$, integrating (30) from $-\infty$ to $\infty$ yields

$$
\begin{aligned}
\int_{-\infty}^{\infty} & Q_{N^{\top}(\zeta) \Sigma N(\eta)}(\ell)(t) d t \\
& =\int_{-\infty}^{\infty} Q_{\Delta}(\ell)(t) d t+Q_{\Xi}(\ell)(\infty)-Q_{\Xi}(\ell)(-\infty) \\
& =\int_{-\infty}^{\infty} Q_{\Delta}(\ell)(t) d t \geq 0 .
\end{aligned}
$$

In the last inequality, we have used the fact that $\Delta \geq 0$.

Recall that compact supportness of $\ell \in D^{-1}\left(\frac{d}{d t}\right) w$ is guaranteed by Proposition 5. Hence, (31) implies that $Q_{\Phi}^{\mathbf{r}}$ is average nonnegative.

Remark 2. We give a remark regarding to $Q_{\Psi}$ and $Q_{\Delta}$ introduced in the proof of sufficiency. Since $Q_{\Delta}(\ell)(t) \geq 0$ holds for all $t \in \mathbb{R}$ and for all $\ell \in \mathfrak{C}^{\infty}\left(\mathbb{R}, \mathbb{R}^{\mathrm{w}}\right),(30)$ reduces to a version of dissipation inequality

$Q_{N^{\top}(\zeta) \Sigma N(\eta)}(\ell)(t) \geq \frac{d}{d t} Q_{\Xi}(\ell)(t) \quad \forall t \in \mathbb{R}, \forall \ell \in D^{-1}\left(\frac{d}{d t}\right) w$.

In this case, $Q_{\Xi}$ and $Q_{\Delta}$ respectively serve as a storage function and a dissipation rate for the QDF $Q_{\Phi}^{\mathbf{r}}$ induced by a rational matrix in $\mathbb{R}_{\mathrm{s}}^{\mathrm{w} \times \mathrm{w}}(\zeta, \eta)$. In other words, the system defined by $v=G\left(\frac{d}{d t}\right) w$, or (13), is dissipative with respective to the supply rate $s=v^{\top} \Sigma v=Q_{N^{\top}(\zeta) \Sigma N(\eta)}(\ell) \in$ $Q_{\Phi}^{\mathbf{r}}(w)$.

The next lemma provides a frequency domain condition for the average nonnegativity.

Lemma 2. Under Assumption 1, there exist $\Psi \in \mathbb{R}_{\mathrm{s}}^{\mathrm{w} \times \mathrm{w}}(\zeta, \eta)$ and $F \in \mathbb{R}^{\bullet \times w}(\xi)$ satisfying (25) if and only if

$$
\Phi(\bar{\lambda}, \lambda) \geq 0 \quad \forall \lambda \in \mathrm{i} \mathbb{R} \backslash\{\text { poles of } G(\xi)\} .
$$

Proof: $(\Rightarrow)$ It readily follows from $(25)$ that

$$
\Phi(\bar{\lambda}, \lambda)=(F(\lambda))^{*} F(\lambda) \geq 0 \quad \forall \lambda \in \mathrm{i} \mathbb{R} \backslash\{\text { poles of } G(\xi)\} .
$$

$(\Leftarrow)$ Let $G(\xi)=N(\xi) D^{-1}(\xi)$ be a right coprime factorization of $G(\xi)$ over $\mathbb{R}[\xi]$. Since $\operatorname{det} D(\lambda) \neq 0$ holds for all $\lambda \in \mathrm{i} \backslash\{$ poles of $G(\xi)\}$, and since $N(\lambda)$ is continuous in $\lambda \in \mathbb{C}$, we obtain

$$
\begin{gathered}
\Phi(\bar{\lambda}, \lambda) \geq 0 \quad \forall \lambda \in \mathrm{i} \mathbb{R} \backslash\{\text { poles of } G(\xi)\} \\
\Leftrightarrow N^{\top}(\bar{\lambda}) \Sigma N(\lambda) \geq 0 \quad \forall \lambda \in \mathrm{i} \mathbb{R} .
\end{gathered}
$$

The latter condition is equivalent to the existence of polynomial matrices $\hat{\Psi} \in \mathbb{R}_{\mathrm{s}}^{\mathrm{w} \times \mathrm{w}}[\zeta, \eta]$ and $\hat{F} \in \mathbb{R}^{\bullet \times{ }^{\mathrm{w}}}[\xi]$ such that

$$
(\zeta+\eta) \hat{\Psi}(\zeta, \eta)+\hat{F}^{\top}(\zeta) \hat{F}(\eta)=N^{\top}(\zeta) \Sigma N(\eta)
$$

(see Proposition 2 (ii) $\Leftrightarrow($ iv)). Thus, we obtain (25) by defining $\Psi(\zeta, \eta)=D^{-\top}(\zeta) \hat{\Psi}(\zeta, \eta) D^{-1}(\eta)$ and $F(\xi)=$ $\hat{F}(\xi) D^{-1}(\xi)$, and by pre- and post-multiplying the above equation by $D^{-\top}(\zeta)$ and $D^{-1}(\eta)$, respectively.

Summarizing the discussions in Lemmas 1 and 2, we obtain the following theorem.

Theorem 1. Under Assumption 1, the following statements are equivalent.

(i) $\int Q_{\Phi}^{\mathbf{r}} \geq 0$ (average nonnegative).

(ii) There exists a symmetric and factorizable rational matrix $\Psi \in \mathbb{R}_{\mathrm{s}}^{\mathrm{w} \times \mathrm{w}}(\zeta, \eta)$ such that

$$
\dot{\Psi}-\Phi \leq 0 .
$$

(iii) $\Phi(\bar{\lambda}, \lambda) \geq 0$ holds for all $\lambda \in \mathrm{i} \mathbb{R} \backslash\{$ poles of $G(\xi)\}$. 
(iv) There exist a symmetric and factorizable rational matrix $\Psi \in \mathbb{R}_{\mathrm{s}}^{\mathrm{W} \times \mathrm{w}}(\zeta, \eta)$ and a rational matrix $F \in$ $\mathbb{R}_{\mathrm{S}}^{\bullet \times \mathrm{w}}(\xi)$ such that

$$
\Phi(\zeta, \eta)=(\zeta+\eta) \Psi(\zeta, \eta)+F^{\top}(\zeta) F(\eta) .
$$

Moreover, among $\Psi$ 's satisfying (33) or (34), there exists one for which $D^{\top}(\zeta) \Psi(\zeta, \eta) D(\eta)$ is a polynomial matrix for the right coprime factors $(N, D)$ of $G(\xi)$.

Proof: It is easily seen from Proposition 8 that $\dot{\Psi}-\Phi \leq 0$ holds iff there exists $F \in \mathbb{R}^{\bullet \times w}(\xi)$ satisfying

$$
\Phi(\zeta, \eta)-(\zeta+\eta) \Psi(\zeta, \eta)=F^{\top}(\zeta) F(\eta)
$$

This implies the equivalence between (ii) and (iv). Also, (i) $\Leftrightarrow($ iv) and (iii) $\Leftrightarrow$ (iv) are obvious from Lemmas 1 and 2, respectively. The existence of $\Psi(\zeta, \eta)$ for which $\dot{\Psi}-\Phi \leq 0$ and $D^{\top}(\zeta) \Psi(\zeta, \eta) D(\eta)$ is a polynomial matrix is also clear from the proof of the sufficiency of Lemma 1.

Remark 3. The equivalence between (iii) and (iv), namely Lemma 2, claims that the para-Hermitian rational matrix $\Phi(-\xi, \xi) \in \mathbb{R}^{\mathrm{w} \times \mathrm{w}}(\xi)$ admits a spectral factorization

$$
\Phi(-\xi, \xi)=F^{\top}(-\xi) F(\xi), \quad F \in \mathbb{R}^{\mathrm{w} \times \mathrm{w}}(\xi),
$$

if and only if the condition (iii) is satisfied (This condition was first proved by Youla (1961)). Hence, the above result provides a behavioral interpretation to Youla's spectral factorization theorem.

\section{Example 1 (continued). Since}

we have

$$
\Phi(-\xi, \xi)=\frac{-R \xi^{2}}{\left(\frac{1}{C}-R \xi+L \xi^{2}\right)\left(\frac{1}{C}+R \xi+L \xi^{2}\right)},
$$

$$
\Phi(\bar{\lambda}, \lambda)=R\left|\frac{\lambda}{\frac{1}{C}+R \lambda+L \lambda^{2}}\right|^{2} \geq 0 \quad \forall \lambda \in \mathrm{i} \mathbb{R} .
$$

Hence, a spectral factor of $\Phi(-\xi, \xi)$ exists, and is given by

It follows that

$$
F(\xi)=\frac{\sqrt{R} \xi}{\frac{1}{C}+R \xi+L \xi^{2}} .
$$

$$
\begin{aligned}
\Psi(\zeta, \eta) & =\frac{\Phi(\zeta, \eta)-F(\zeta) F(\eta)}{\zeta+\eta} \\
& =\frac{\frac{1}{2 C}+\frac{1}{2} L \zeta \eta}{\left(\frac{1}{C}+R \zeta+L \zeta^{2}\right)\left(\frac{1}{C}+R \eta+L \eta^{2}\right)} .
\end{aligned}
$$

Since the right coprime factors of $G(\xi)$ are given by

$$
\begin{aligned}
& D(\xi)=\frac{1}{C}+R \xi+L \xi^{2}, \\
& N(\xi)=\frac{1}{2}\left(\begin{array}{l}
D(\xi)+\xi \\
D(\xi)-\xi
\end{array}\right)=\frac{1}{2}\left(\begin{array}{c}
\frac{1}{C}+(R+1) \xi+L \xi^{2} \\
\frac{1}{C}+(R-1) \xi+L \xi^{2}
\end{array}\right),
\end{aligned}
$$

the polynomial matrices in $(28),(29)$ are given by

$$
\begin{aligned}
& \Xi(\zeta, \eta)=D(\zeta) \Psi(\zeta, \eta) D(\eta)=\frac{1}{2 C}+\frac{1}{2} L \zeta \eta, \\
& \Delta(\zeta, \eta)=D(\zeta) F(\zeta) F(\eta) D(\eta)=R \zeta \eta
\end{aligned}
$$

In addition, the observable (polynomial) image representation of $v=G\left(\frac{d}{d t}\right) w$ is given by

$$
\left(\begin{array}{c}
v \\
w
\end{array}\right)=\left(\begin{array}{c}
\frac{1}{2}(V+I) \\
\frac{1}{2}(V-I) \\
-V^{--}
\end{array}\right)=\left(\begin{array}{c}
N\left(\frac{d}{d t}\right) \\
\hdashline \bar{D}\left(\frac{d}{d t}\right)
\end{array}\right) q .
$$

Note that, in this case, the latent variable is exactly the electrical charge $q$. Hence, by Remark $2, \Xi(\zeta, \eta)$ and
$\Delta(\zeta, \eta)$ induce the storage function and the dissipation rate as

$$
Q_{\Xi}(q)=\frac{1}{2 C} q^{2}+\frac{1}{2} L I^{2}, \quad Q_{\Delta}(q)=R I^{2}, \quad I=\frac{d q}{d t} .
$$

In fact, the first and second terms of $Q_{\Xi}(q)$ represent the energies stored in the capacitor and the inductor, respectively.

Next, we consider the half-line nonnegativity of $Q_{\Phi}^{\mathrm{r}}$.

Definition 8. Under Assumption 1, $Q_{\Phi}^{\mathbf{r}}$ is called half-line nonnegative. denoted by $\int^{t} Q_{\Phi}^{\mathbf{r}} \geq 0$, if there holds

$$
\int_{-\infty}^{0} s(t) d t \geq 0 \quad \forall s \in \hat{Q}_{\Phi}^{\mathrm{r}}(w), \forall w \in \mathfrak{C}^{\infty}\left(\mathbb{R}, \mathbb{R}^{\mathrm{w}}\right) \cap \mathfrak{D} .
$$

The half-line nonpositivity is also defined in the same way.

Theorem 2. Under Assumption 1, the following statements are equivalent.

(i) $\int^{t} Q_{\Phi}^{\mathbf{r}} \geq 0$ (half-line nonnegative).

(ii) There exists a symmetric and factorizable rational matrix $\Psi \in \mathbb{R}_{\mathrm{s}}^{\mathrm{w} \times \mathrm{w}}(\zeta, \eta)$ such that

$$
\dot{\Psi}-\Phi \leq 0 \quad \& \quad \Psi \geq 0 \text {. }
$$

(iii) There exist a symmetric and factorizable $\Psi \in$ $\mathbb{R}_{\mathrm{s}}^{\mathrm{w} \times \mathrm{w}}(\zeta, \eta)$ and a rational matrices $F, K \in \mathbb{R}^{\bullet \times \mathrm{w}}(\xi)$ satisfying (34) and

$$
\Psi(\zeta, \eta)=K^{\top}(\zeta) K(\eta)
$$

Moreover, among $\Psi$ 's satisfying (36), there exists one for which $D^{\top}(\zeta) \Psi(\zeta, \eta) D(\eta)$ is a polynomial matrix for the right coprime factors $(N, D)$ of $G(\xi)$.

We omit the proof, since the theorem can be proved almost in the same way as Theorem 1 .

We have the same observation as Remark 2 that, for $\Psi(\zeta, \eta)$ satisfying the conditions in Theorem 2, the polynomial QDF $Q_{\Xi}(\ell), \Xi(\zeta, \eta)=D^{\top}(\zeta) \Psi(\zeta, \eta) D(\eta)$ serves as a "nonnegative" storage function for the system defined by $v=G\left(\frac{d}{d t}\right) w$ and the supply rate $s=v^{\top} \Sigma v \in Q_{\Phi}^{\mathbf{r}}(w)$.

\section{CONCLUDING REMARKS}

In this paper, we have presented a new and more general formulation of a QDF in terms of rational functions rather than polynomials. It has turned out that the rational QDF defines a set unlike the polynomial QDF. We have also shown that the notions of nonnegativity, average nonnegativity and half-line nonnegativity can be generalized for the rational QDF, and have derived their necessary and sufficient conditions. It remains as future topics to study the rational QDF along a given behavior and its application to the Lyapunov stability and the dissipation theory. It should be noted that the rational QDF has many important applications in system and control theory: for example, the dissipation theory for linear systems defined by rational representations, the stability analysis of interconnected systems with rational multipliers, and so forth. 


\section{REFERENCES}

M.N. Belur and H.L. Trentelman. The strict dissipativity synthesis problem and the rank of the coupling QDF. Syst. Contr. Letters, 51(3-4): 247-258, 2004.

T. Iwasaki and S. Hara. Well-posedness of feedback systems: insights into exact robustness analysis and approximate computations. IEEE Trans. Automat. Contr. 43(5): 619-630, 1998.

O. Kaneko and T. Fujii. Discrete-time average positivity and spectral factorization in a behavioral framework. Syst. Contr. Letters, 39(1): 31-44, 2000.

O. Kaneko and T. Fujii. When is a storage function a state function in discrete time? SIAM J. Contr. Optimiz., 42(4): 1374-1394, 2003.

O. Kaneko and T. Fujii. The behavioral approach to dissipation theory of dynamical systems: Based on QDF's. Syst. Contr. Inform., 48(5): 171-177, 2004 (in Japanese).

C. Kojima and K. Takaba. A generalized Lyapunov stability theorem for discrete-time systems based on quadratic difference forms. Proc. 44th IEEE Conf. Decis. Contr., and Eur. Contr. Conf. 2005 (CDC-ECC2005), pages 2911-2916, 2005.

A. Megretski and A. Rantzer. System analysis via integral quadratic constraints. IEEE Trans. Automat. Contr., 42(6): 819-830, 1997.

R. Peeters and P. Rapisarda. A two-variable approach to solve the polynomial Lyapunov equation. Syst. Contr. Letters, 42(2): 117-126, 2001.

I. Pendharkar and H. Pillai. Systems with sector bound nonlinearities: A behavioral approach. Syst. Contr. Letters, 72(2): 112-122, 2007.

J.W. Polderman and J.C. Willems. Introduction to Mathematical Systems Theory: A Behavioral Approach, Springer-Verlag, 1998.

K. Takaba. Robust stability analysis of uncertain interconnection in the behavioral framework. Proc. of 16th IFAC World Congress, Prague, paper code: Th-A13$\mathrm{TO} / 3,2005$.

H.L. Trentelman and J.C. Willems. $H^{\infty}$ control in a behavioral context: The full information case. IEEE Trans. Automat. Contr., 44(3): 521-536, 1999.

J.C. Willems and H.L. Trentelman. On quadratic differential forms. SIAM J. Contr. Optimiz., 36(5): 1703-1749, 1998.

J.C. Willems and H.L. Trentelman. Synthesis of dissipative systems using quadratic differential forms: Parts I and II. IEEE Trans. Automat. Contr., 47(1): 53-86, 2002.

J.C. Willems and M.E. Valcher. Linear-quadratic control and quadratic differential forms. Proc. of 16th IFAC World Congress, Prague, Paper code: Mo-A15-TO/1, 2005.

J.C. Willems and K. Takaba. Dissipativity and stability of interconnections. Int. J. of Robust and Nonlinear Control, 17: 563-586, 2007.

J.C. Willems and Y. Yamamoto. Behaviors defined by rational functions. Linear Algebra and its Applications, 425(3-4): 226-24, 2007.

D.C. Youla. On the factorization of rational matrices. IRE Trans. Inform. Theory, 7: 172-189, 1961. 